\title{
A NUMERICAL UPPER BOUND FORMULATION WITH SENSIBLY-ARRANGED VELOCITY DISCONTINUITIES AND ORTHOTROPIC MATERIAL STRENGTH BEHAVIOUR
}

\author{
Minguing Li, Josef Füssl, Markus Lukacevic, Josef Eberhardsteiner \\ Vienna University of Technology, Vienna, Austria \\ e-mail: mingjing.li@tuwien.ac.at
}

Christopher M. MARTin

University of Oxford, Oxford, UK

\begin{abstract}
Numerical limit analysis allows for fast estimates of the collapse load of structures exhibiting ideal plastic material behaviour. In numerical upper bound formulations, the description of the unknown velocity field can be extended by introducing velocity discontinuities between finite elements. Through these additional degrees of freedom, localised failure modes may be approximated more accurately and better upper bounds can be obtained. In the existing formulations, such discontinuities are typically introduced between all elements and the description is restricted to isotropic failure behaviour. In this work, a general 3D upper bound formulation is briefly proposed, allowing the consideration of both isotropic and orthotropic yield functions within finite elements as well as at velocity discontinuities. The concept of "projecting" a stress-based orthotropic yield function onto a certain discontinuity is presented, giving a traction-based yield function which allows for a consistent description of the material strength behaviour across the interface. The formulation is verified by means of two classical examples, the rigid strip footing and the block with asymmetric holes. Furthermore, based on the computation of potential orientations of plastic flow localisation, a simple concept for a sensible arrangement of velocity discontinuities is proposed. It is shown that this concept performs very well for isotropic as well as anisotropic material strength behaviour. A feature of the present work is that, velocity jumps are allowed only across the prescribed finite element interfaces determined from the sensible discontinuity arrangement. Good upper bounds similar to those in the existing works are obtained with far fewer degrees of freedom.
\end{abstract}

Keywords: numerical upper bound formulations, localised failure modes, traction-based yield function, sensible arrangement of velocity discontinuities, orthotropic material strength behaviour

\section{Introduction}

\subsection{Numerical limit analysis}

The main objective of limit analysis is the determination of load bearing capacities of structures exhibiting an elastoplastic material response. To achieve this, limit analysis concentrates on the critical energy dissipation rate at the time instant of structural failure, and the basic task can be expressed as follows: Find the kinematically admissible velocity field which minimises energy dissipation over the set of all statically admissible stress fields which maximise the dissipated energy (Ciria et al., 2008). Statically admissible stress fields are required to be in equilibrium, fulfil the static boundary conditions and obey a plastic yield criterion at each point of the body. Kinematically admissible velocity fields are subject to compatibility, the kinematic 
boundary conditions, and fulfil an associated plastic flow rule at each point of the body. Unfortunately, the so-defined saddle-point problem can be solved only for simple geometric and loading situations as well as for simple material behaviours. In more complex situations, the plastic flow compatibility in the so-called static principle or the static equilibrium in the so-called kinematic principle may be relaxed, providing lower and upper bounds on the exact load bearing capacity of a structure according to the bounding theorems by Drucker et al. $(1951,1952)$.

However, for complex problems, the application of these bounding theorems (in the context of limit analysis) in an analytical way is very limited and often not possible. Thus, finite-element-based formulations were first introduced in the 1970s (Belytschko and Hodge, 1970; Lysmer, 1970; Anderheggen and Knöpfel, 1972; Maier et al., 1972), and gained popularity from then on. The computational efficiency and accuracy of such numerical formulations strongly depend on the mathematical programming method used to solve the underlying optimisation problems. At the early stage, the limit analysis theorems were formulated as linear optimisation problems, by linearising the applied plastic yield functions. At the turn of the millennium, Lyamin and Sloan (2002a,b) proposed more general lower and upper bound formulations allowing for nonlinear yield functions, which were solved using nonlinear programming concepts. However, local smoothing was required for yield functions with singularities, e.g. the Mohr-Coulomb yield function. Subsequently, during the past two decades, second-order cone programming (SOCP) has been proven to be an excellent alternative method by Makrodimopoulos and Martin (2006, 2007) and Ciria and Peraire (2004) for cohesive-frictional materials and Füssl et al. (2008) for composite materials, with sufficient robustness and efficiency to solve large-scale nonlinear optimisation problems of numerical limit analysis. Such implementations allow the applications of many different yield functions in their native form, since most of the commonly-used yield functions can be formulated as second-order cones. In this work, SOCP is employed to solve the nonlinear optimisation problems arising from the presented limit analysis formulations.

The efficiency and accuracy of such formulations is also strongly influenced by the chosen finite elements and related shape functions. In order to obtain rigorous upper bound solutions, for example, the associated plastic flow rule must be satisfied throughout the whole body. Basically, this can be achieved by using constant strain triangular elements, which are often combined with velocity discontinuities between element boundaries (Bottero et al., 1980; Sloan and Kleeman, 1995; Lyamin and Sloan, 2002b). To improve the quality of upper bound solutions, the use of higher order interpolation functions is desired. Makrodimopoulos and Martin (2007) showed that the associated plastic flow rule could also be enforced throughout the whole body by using linear strain triangular elements, leading to a better performance than constant strain elements even without discontinuities. As a further development, the meshless method was implemented for numerical upper bound approaches in (Le et al., 2010; Liu and Zhao, 2013; Yu et al., 2016). However, in such implementations with high order shape functions, it is difficult to guarantee both compatibility and satisfaction of the associated plastic flow rule throughout each element.

Additionally or as an alternative to the use of high order elements, velocity discontinuities can be implemented in upper bound formulations to increase their effectiveness. In (Chen et al., 2003; Milani and Lourenço, 2009), for example, rigid elements were used and plastic dissipation was only allowed between finite elements. Such approaches are highly dependent on the mesh and even adaptive mesh refinement cannot fully compensate for this issue. An approach without using classical finite elements is the so-called discontinuity layout optimisation (DLO), where velocity discontinuities are determined by using a truss layout optimisation algorithm based on a prescribed grid (Smith and Gilbert, 2007; Hawksbee et al., 2013). This approach performs well for $2 \mathrm{D}$ problems, but the determination of complex failure mechanisms in $3 \mathrm{D}$ bodies requires a fine grid and large computational effort.

For this reason, in the authors' opinion, the most promising approach so far to obtain rigorous upper bound solutions still seems to be the use of solid finite elements with or without velocity 
discontinuities. In Krabbenhøft et al. (2005) zero-thickness interface elements between constant strain elements are introduced, which perform well for a large number of applications. Another development can be found in Makrodimopulous and Martin (2008), where velocity discontinuities are implemented between linear strain elements. In order to increase the efficiency of the upper bound formulations, adaptive mesh refinement was introduced by Ciria and Peraire (2004), Ciria et al. (2008) and Martin (2011). However, a targeted arrangement of discontinuities, as will be proposed in this work, has not been introduced until now.

\subsection{Objective of the paper}

In several previous works, e.g. in (Füssl et al., 2017; Li et al., 2018), anisotropic yield functions have been implemented in numerical upper bound formulations. To the authors' knowledge, the combination of anisotropic yield functions and velocity discontinuities has not previously been presented, although it could significantly improve the capability of upper bound approaches in handling localised plastic failure for anisotropic materials, like wood or fibre reinforced composites. In particular, it is beneficial if the alignment of discontinuities is tuned to the direction of localised plastic failure.

Thus, the main objectives of this work can be introduced as follows:

1. The formulation of 3D numerical upper bound approaches with anisotropic yield functions, quadratic shape functions for the velocity fields, and velocity discontinuities.

2. To allow for a consistent description of plastic failure also across velocity discontinuities, the derivation of a traction-based yield function which is in accordance with the stress-based yield function assigned to the solid finite elements/bulk material.

3. Implementation of an initial concept for a sensible introduction and arrangement of velocity discontinuities only in failure regions.

According to these objectives, the paper is structured as follows. A quite general numerical upper bound approach is briefly proposed in Section 2, able to consider plastic energy dissipation in both finite elements and discontinuities obeying an anisotropic failure criterion. Furthermore, the process for obtaining the required traction-based yield functions for the discontinuities is described. A verification of the implemented upper bound formulations by means of well-known examples can be found in Section 3, as well as a discussion about the performance of velocity discontinuities. Finally, a brief summary and concluding comments are given in Section 4 .

\section{Upper bound approaches}

The upper bound theorem focuses exclusively on the kinematically admissible velocity fields $\dot{\mathbf{u}}=\left(\dot{u}_{x}, \dot{u}_{y}, \dot{u}_{z}\right)^{\mathrm{T}} \in \mathfrak{R}^{3}$, and by minimising the internal plastic energy dissipation rate $W_{\text {int }}$, which has to be equal to the work rate of the external loads $W_{\text {ext }}$, the resulting failure state provides an upper bound for the exact collapse load. A kinematically admissible velocity field $\dot{\mathbf{u}}$ has to satisfy the compatibility, the associated plastic flow rule, and the kinematic boundary conditions at each point of the considered body. Additionally, a velocity-jump field $\Delta \dot{\mathbf{u}}=\left(\Delta \dot{u}_{x}, \Delta \dot{u}_{y}, \Delta \dot{u}_{z}\right)^{\mathrm{T}} \in \mathfrak{R}^{3}$ is introduced, describing localised interface plastic failure across an prescribed interior surface.

The internal energy dissipation rate $W_{\text {int }}$ is composed of a part referring to material failure in the continuum body $\Omega$ and a part related to the energy dissipation at interior surfaces $\Gamma^{d i s}$, and reads

$$
W_{\text {int }}=\int_{\Omega} d_{p}^{m a t}(\dot{\boldsymbol{\varepsilon}}) d V+\int_{\Gamma^{d i s}} d_{p}^{d i s}(\Delta \dot{\mathbf{u}}) d A
$$


with the plastic dissipation functions

$$
\begin{array}{lll}
d_{p}^{\text {mat }}=\sup _{\boldsymbol{\sigma} \in \mathcal{F}} \boldsymbol{\sigma}^{\mathrm{T}} \dot{\boldsymbol{\varepsilon}} & \mathcal{F}=\{\boldsymbol{\sigma} \mid f(\boldsymbol{\sigma}) \leqslant 0\} & \text { in } \Omega \\
d_{p}^{d i s}=\sup _{\mathbf{t} \in \mathcal{D}} \mathbf{t}^{\mathrm{T}} \Delta \dot{\mathbf{u}} & \mathcal{D}=\{\mathbf{t} \mid f(\mathbf{t}) \leqslant 0\} & \text { on } \Gamma^{\text {dis }}
\end{array}
$$

where $\dot{\varepsilon}=\left(\dot{\varepsilon}_{x x}, \dot{\varepsilon}_{y y}, \dot{\varepsilon}_{z z}, \dot{\varepsilon}_{x y}, \dot{\varepsilon}_{y z}, \dot{\varepsilon}_{x z}\right)^{\mathrm{T}} \in \mathfrak{R}^{6}$ represents the plastic strain-rate field, $\boldsymbol{\sigma}=\left(\sigma_{x x}, \sigma_{y y}, \sigma_{z z}, \tau_{x y}, \tau_{y z}, \tau_{x z}\right)^{\mathrm{T}} \in \mathfrak{R}^{6}$ the stress field and $\mathbf{t}=\left(t_{x}, t_{y}, t_{z}\right)^{\mathrm{T}} \in \mathfrak{R}^{3}$ the surface traction field. $f(\boldsymbol{\sigma}) \leqslant 0$ and $f(\mathbf{t}) \leqslant 0$ denote the stress-based yield function for $\Omega$ and the traction-based yield function for $\Gamma^{\text {dis }}$, respectively.

The upper bound theorem can then be formulated as a nonlinear optimisation problem, reading

$$
\begin{array}{lll}
\min & W_{\text {int }} & \\
\text { s.t. } & \dot{\boldsymbol{\varepsilon}}=\operatorname{div} \dot{\mathbf{u}} & \text { in } \Omega \\
& \dot{\mathbf{u}}=\dot{\mathbf{u}}_{b} & \text { on } \Gamma \\
& \dot{\boldsymbol{\varepsilon}}=\dot{\lambda}_{\sigma} \partial f(\boldsymbol{\sigma}) / \partial \boldsymbol{\sigma} & \text { in } \Omega \\
& \Delta \dot{\mathbf{u}}=\dot{\lambda}_{t} \partial f(\mathbf{t}) / \partial \mathbf{t} & \text { on } \Gamma^{\text {dis }}
\end{array}
$$

in which the constraints enforce compatibility between the velocities and the plastic strain-rates, the kinematic boundary conditions, and the associated plastic flow rule both in the continuum $\Omega$ and at the interior surfaces $\Gamma^{d i s}$. In the second constraint, $\dot{\mathbf{u}}_{b}$ refers to the prescribed velocity boundary conditions defined over the whole surface $\Gamma=\partial \Omega$ of the continuum body. In the last two constraints, $\dot{\lambda}_{\sigma}$ and $\dot{\lambda}_{t}$ are plastic multipliers, determining the magnitude of plastic flow within the continuum and at the discontinuities, respectively. Note that these two associated plastic flow constraints in Eq. (2.3) are valid only when the yield function is differentiable everywhere. If singular apex points exist, additional technology is required, and the use of SOCP in this work ensures that such points are handled naturally.

As shown in Makrodimopoulos and Martin (2007), using the duality of nonlinear programming, a mathematically equivalent optimisation problem to Eq. (2.3) can be formulated, reading

$$
\begin{array}{lll}
\max & W_{\text {ext }} \\
\text { s.t. } & \int_{\Omega}(\operatorname{div} \dot{\mathbf{u}})^{\mathrm{T}} \boldsymbol{\sigma} d V+\int_{\Gamma^{d i s}} \Delta \dot{\mathbf{u}}^{\mathrm{T}} \mathbf{t} d A=\int_{\Omega} \dot{\mathbf{u}}^{\mathrm{T}} \beta \mathbf{g} d V+\int_{\Gamma} \dot{\mathbf{u}}^{\mathrm{T}} \beta \mathbf{t} d A & \text { in } \Omega \\
& f(\boldsymbol{\sigma}) \leqslant 0 & \text { in } \Omega \\
& f(\mathbf{t}) \leqslant 0 & \text { on } \Gamma^{\text {dis }}
\end{array}
$$

in which the first constraint represents weak equilibrium of the dissipated energy, and the objective function is related to the external work rate given as

$$
W_{e x t}=\int_{\Omega} \dot{\mathbf{u}}^{\mathrm{T}} \beta \mathbf{g} d V+\int_{\Gamma} \dot{\mathbf{u}}^{\mathrm{T}} \beta \mathbf{t} d A
$$

where $\beta$ denotes a load multiplier applied to the surface traction field $\mathbf{t}$ and the prescribed body force field $\mathrm{g} \in \mathfrak{R}^{3}$.

For the discretisation of the upper bound optimisation problem, tetrahedral linear-strain simplex elements are used, as introduced for the 2D upper bound problem under plane strain conditions in Makrodimopoulos and Martin $(2007,2008)$ and for the 3D upper bound problem in Martin and Makrodimopoulos (2008). Thus, the velocity field is approximated using quadratic interpolation functions and the plastic strain-rate field is described by linear shape functions. Worth mentioning is that each finite element has its own strain-rate evaluation nodes, which means that adjacent nodes from different elements share the same coordinates but can have different 
strain-rate states. The exact representation of this approximation is given in Makrodimopoulos and Martin (2008). In this work, to assess the capability of the discontinuity arrangement, velocity jumps are allowed only across particular prescribed finite element interfaces determined by the arrangement, on which adjacent elements have their own velocity evaluation nodes.

Finally, as introduced in detail by the authors in Li et al. (2018), the discretised formulation of the dual upper bound optimisation problem, Eq. (2.4), can be written as

$$
\begin{aligned}
& \max W_{\text {ext }} \\
& \text { s.t. } \quad \mathbf{A}_{U B}^{m a t}{ }^{\mathrm{T}} \hat{\mathbf{q}}_{\sigma}+\mathbf{A}_{U B}^{d i s} \widehat{\mathbf{q}}_{t}^{d i s}=\beta \mathbf{A}_{U B}^{b c} \widehat{\mathbf{q}}_{t}^{b c} \\
& \widehat{\mathbf{s}}_{\sigma}^{m a t, i}=\widehat{\mathbf{a}}_{\sigma}^{m a t, i}+\mathbf{B}_{\sigma}^{m a t} \mathbf{R}_{\dot{\varepsilon}}^{m a t} \widehat{\mathbf{q}}_{\sigma}^{m a t, i} \\
& \widehat{\mathbf{s}}_{\sigma}^{m a t, i} \in \mathscr{C} \\
& \widehat{\mathbf{s}}_{t}^{d i s, j}=\widehat{\mathbf{a}}_{t}^{d i s, j}+\mathbf{B}_{t}^{d i s} \mathbf{R}_{t}^{d i s} \widehat{\mathbf{q}}_{t}^{d i s, j} \\
& \widehat{\mathbf{s}}_{t}^{d i s, j} \in \mathscr{C}
\end{aligned}
$$

with the matrices $\mathbf{A}_{U B}$ obtained by applying the linear compatibility operator to the related shape functions of the velocity (or the velocity jump), within the finite elements (mat), at the discontinuities between elements (dis), and at the boundary $(b c)$. The vectors $\widehat{\mathbf{q}}_{\sigma}$, $\widehat{\mathbf{q}}_{t}^{d i s}$, and $\widehat{\mathbf{q}}_{t}^{b c}$ collect all nodal degrees of freedom related to stress-like quantities and surface tractions. Note that, for an arbitrary vector $\mathbf{x}$, the symbol $\widehat{\mathbf{x}}=\int_{\Omega_{e}} \mathbf{x} d V$ refers to the volume-integrated quantity over each element. The remaining constraints represent a second-order cone formulation of a general quadratic yield function for both the solid material (mat) and the discontinuities (dis), with $i$ and $j$ ranging from 1 to the number of stress and traction evaluation nodes, respectively. Thereby, the matrices $\widehat{\mathbf{a}}$ and $\mathbf{B}$ contain strength parameters and the matrices $\mathbf{R}$ represent transformation operators, rotating the stress tensors into the principal material direction and the surface traction vector into the direction of the corresponding discontinuity. The external work rate in discretised form can be written as

$$
W_{e x t}=\sum_{b c=1}^{U B C} \sum_{i=1}^{6} \beta \mathbf{q}_{\dot{i}, l o c}^{b c, i} \mathrm{q}_{t, l o c}{ }^{b c, i}
$$

where $U B C$ denotes the number of 6-noded boundary surface triangular elements with a prescribed local traction field $\widehat{\mathbf{q}}_{t, l o c}^{b c}$ and $\mathbf{q}_{i, l o c}^{b c}$ represents the related velocity degrees of freedom.

In previous upper bound formulations (Sloan and Kleeman, 1995; Krabbenhoft et al., 2005; Makrodimopoulos and Martin, 2008) only isotropic yield functions based on shear failure mechanisms, e.g. the von Mises or the Mohr-Coulomb yield function, were considered, leading to a straightforward definition of failure at the discontinuities between elements. On the contrary, in the upper bound formulation Eq. (2.6), the quite general orthotropic yield function according to Tsai-Wu can be implemented, reading

$$
\mathbf{q}^{i \mathrm{~T}} \mathbf{P} \mathbf{q}^{i}+\left(\frac{1}{2} \mathbf{F}^{+\mathrm{T}} \mathbf{q}^{i}\right)^{2}-\left(1-\frac{1}{2} \mathbf{F}^{-\mathrm{T}} \mathbf{q}^{i}\right)^{2} \leqslant 0
$$

with $i$ as the evaluation point of $\mathbf{q}$ either for the stress field (with subscript $\sigma$ ) in the element or for the traction field (with subscript $t$ ) at a discontinuity. The vectors $\mathbf{F}^{+}, \mathbf{F}^{-}$and matrix $\mathbf{P}$ are related to the terms in Eq. (2.6) as follows

$$
\mathbf{a}=\left[\begin{array}{l}
1 \\
\mathbf{0}
\end{array}\right] \quad \mathbf{B}=\left[\begin{array}{c}
-\frac{1}{2} \mathbf{F}^{-\mathrm{T}} \\
\mathbf{D} \\
\frac{1}{2} \mathbf{F}^{+\mathrm{T}}
\end{array}\right]
$$


where $\mathbf{D}$ is the decomposed product of $\mathbf{P}=\mathbf{D}^{\mathrm{T}} \mathbf{D}$. Note that the matrix dimensions are $\mathbf{F}_{\sigma}^{+} \in \mathfrak{R}^{6}$, $\mathbf{F}_{\sigma}^{-} \in \mathfrak{R}^{6}, \mathbf{P}_{\sigma} \in \mathfrak{R}^{6 \times 6}, \mathbf{D}_{\sigma} \in \mathfrak{R}^{6 \times 6}$ for the stress-based yield function and $\mathbf{F}_{t}^{+} \in \mathfrak{R}^{3}, \mathbf{F}_{t}^{-} \in \mathfrak{R}^{3}$, $\mathbf{P}_{t} \in \mathfrak{R}^{3 \times 3}, \mathbf{D}_{t} \in \mathfrak{R}^{3 \times 3}$ for the traction-based yield function.

In the above, it is assumed that there exists a traction-based yield function for the discontinuities which can also be formulated as a second-order cone. Additionally, it needs to be consistent with the stress-based Tsai-Wu criterion to allow for the description of a homogeneous strength distribution within a body. Since a surface traction state within a discontinuity cannot directly be related to a unique 3D stress state at a material point, the derivation of such a tractionbased yield function is not straightforward. However, according to Wu and Cervera (2014), we can assume a 3D plastic strain-rate state to be localised with respect to a certain discontinuity if the following constraints are satisfied, for orthotropic yield functions which are differentiable everywhere (e.g. Tsai-Wu yield function):

$$
\begin{aligned}
& \Lambda_{m m}^{\text {dis }}\left(\boldsymbol{\sigma}_{\text {loc }}^{\text {dis }}\right)=\frac{\dot{\varepsilon}_{m m}}{\dot{\lambda}_{\sigma}}=\frac{\partial f\left(\boldsymbol{\sigma}_{\text {loc }}^{\text {dis }}, \text { strength par. }\right)}{\partial \sigma_{m m}^{d i s}}=0 \\
& \Lambda_{p p}^{\text {dis }}\left(\boldsymbol{\sigma}_{\text {loc }}^{\text {dis }}\right)=\frac{\dot{\varepsilon}_{p p}}{\dot{\lambda}_{\sigma}}=\frac{\partial f\left(\boldsymbol{\sigma}_{\text {loc }}^{\text {dis }}, \text { strength par. }\right)}{\partial \sigma_{p p}^{\text {dis }}}=0 \\
& \Lambda_{m p}^{\text {dis }}\left(\boldsymbol{\sigma}_{\text {loc }}^{\text {dis }}\right)=\frac{\dot{\varepsilon}_{m p}}{\dot{\lambda}_{\sigma}}=\frac{\partial f\left(\boldsymbol{\sigma}_{\text {loc }}^{\text {dis }}, \text { strength par. }\right)}{\partial \tau_{m p}^{\text {dis }}}=0
\end{aligned}
$$

where $\boldsymbol{\sigma}_{l o c}^{\text {dis }}$ denotes a 3D stress state at a discontinuity with the local coordinate basis $(n-m-p)$ with the normal vector of the discontinuity pointing in the $n$-direction. Note that each of the constraints in Eq. (2.10) can be formulated as a function of the local stress field $\boldsymbol{\sigma}_{\text {loc }}^{\text {dis }}$. By reformulating Eq. (2.10) using the definition of the Cauchy stress tensor, giving $t_{n}^{d i s}=\sigma_{n n}^{d i s}$, $t_{m}^{d i s}=\tau_{n m}^{d i s}, t_{p}^{d i s}=\tau_{n p}^{d i s}$, the three remaining stress tensor components $\sigma_{m m}^{d i s}, \sigma_{p p}^{d i s}$, and $\tau_{m p}^{d i s}$ can be expressed as functions, hereafter referred to as $L^{d i s}$, of $t_{n}^{d i s}, t_{m}^{d i s}, t_{p}^{d i s}$, and certain strength parameters, reading

$$
\begin{aligned}
& \sigma_{m m}^{d i s}=L_{m m}^{d i s}\left(\mathbf{t}_{l o c}^{\text {dis }}, \text { strength par. }\right) \quad \sigma_{p p}^{d i s}=L_{p p}^{d i s}\left(\mathbf{t}_{\text {loc }}^{\text {dis }}, \text { strength par. }\right) \\
& \sigma_{m p}^{\text {dis }}=L_{m p}^{d i s}\left(\mathbf{t}_{\text {loc }}^{\text {dis }}, \text { strength par. }\right)
\end{aligned}
$$

Therefrom, a relationship between the local stress field and the local traction field on $\Gamma_{d i s}$, $\boldsymbol{\sigma}_{l o c}^{d i s}=\mathbf{L}_{t}^{\text {dis }} \mathbf{t}_{\text {loc }}^{\text {dis }}$, under the condition of plastic strain localisation, can be derived. Finally, by making use of this relationship, it is possible to "project" the stress-based formulation of the Tsai-Wu yield function $f\left(\boldsymbol{\sigma}_{l o c}^{\text {dis }}\right) \leqslant 0$ onto a discontinuity, delivering a consistent traction-based yield function $f\left(\mathbf{t}_{l o c}^{\text {dis }}\right) \leqslant 0$.

The main focus of this work is the performance assessment of this approach and to point out how such an approach could be utilised for future concepts of numerical limit analysis. For this reason, in the next Section, several numerical examples are presented and discussed in detail.

\section{Numerical results}

In this Section, numerical results obtained using the proposed upper bound formulation with selectively activated velocity discontinuities are discussed. Two benchmark problems with isotropic yield functions are used for basic verification of the presented approaches. By means of further examples, it is demonstrated that orthotropic plastic failure can also be handled appropriately. Moreover, it will be shown that through the introduction of velocity discontinuities across properly-arranged prescribed interfaces, high-quality upper bound results can be obtained with relatively coarse meshes and, thus, very efficiently. Note that, for convenience, the upper bound 
results obtained using formulations, with and without velocity discontinuities, are referred to as continuous and discontinuous upper bound results, respectively.

All computations presented in the following have been performed on a Linux desktop machine with an AMD Phenom(tm) II X6 1090T CPU (6 cores) and 8GB of RAM. The commercial software package Abaqus was used for mesh generation, but all other pre- and post-processing tasks as well as the assembly of the SOCP optimisation problems were carried out by self-written codes in Fortran. The SOCP optimisation problems themselves were solved using the commercial software MOSEK (2014), which is based on the conic interior-point algorithm described in Andersen et al. (2003).

\subsection{Rigid strip footing}

The rigid strip footing problem, as illustrated in Fig. 1a, with a weightless purely cohesive material is a common benchmark for limit analysis approaches. Upon the assumption of material failure according to Tresca, $\tau \leqslant c$, the ultimate load can be obtained by the classical Prandtl solution $N_{c}^{r e f}=P_{l i m} / c=2+\pi$ (Prandtl, 1920), where $N_{c}$ is the bearing capacity factor, $P_{\text {lim }}$ the collapse load limit, $\tau$ the principal shear stress, and $c$ the coefficient of cohesion. Under plane strain conditions, the Tresca yield function is identical to the von Mises yield function $\sqrt{J_{2}} \leqslant c$, with $J_{2}$ as the second deviatoric stress invariant.
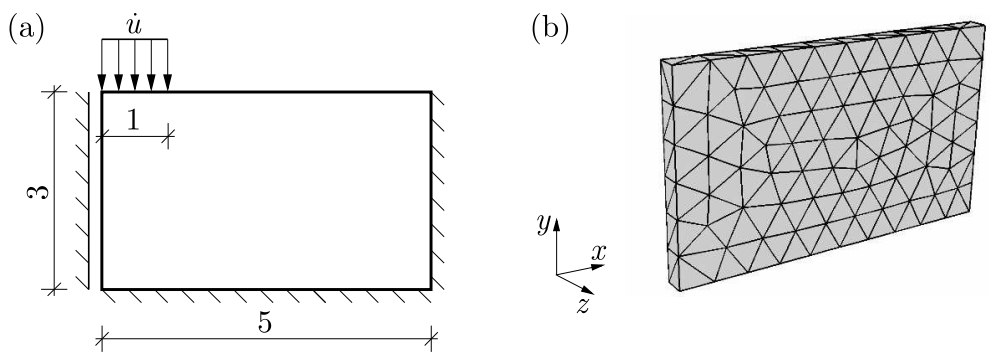

(c)

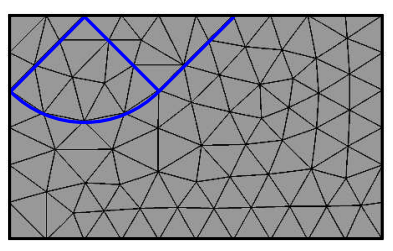

Fig. 1. Rigid strip footing benchmark example: (a) geometry and boundary conditions; (b) example 3D model and illustrative discretisation with 419 elements; (c) the prescribed interfaces for velocity jumps according to Prandtl's failure mechanism (445 elements)

Using the yield function formulation according to Eq. (2.8), the von Mises criterion is defined through

$$
\mathbf{P}_{\sigma}^{\text {mat }}=\frac{1}{3 c^{2}}\left[\begin{array}{cccccc}
1 & -0.5 & -0.5 & 0 & 0 & 0 \\
-0.5 & 1 & -0.5 & 0 & 0 & 0 \\
-0.5 & -0.5 & 1 & 0 & 0 & 0 \\
0 & 0 & 0 & 3 & 0 & 0 \\
0 & 0 & 0 & 0 & 3 & 0 \\
0 & 0 & 0 & 0 & 0 & 3
\end{array}\right] \quad \mathbf{F}_{\sigma}^{\text {mat, }},=\mathbf{F}_{\sigma}^{\text {mat, }-}=\mathbf{0}
$$

A consistent traction-based yield function is easily obtained (Makrodimopoulos and Martin, 2008) by applying the shear strength as the tangential strength at discontinuities, giving

$$
\mathbf{P}_{t}^{d i s}=\frac{1}{c^{2}}\left[\begin{array}{ccc}
0 & 0 & 0 \\
0 & 1 & 0 \\
0 & 0 & 1
\end{array}\right] \quad \mathbf{F}_{t}^{d i s,+}=\mathbf{F}_{t}^{d i s,-}=\mathbf{0}
$$

The geometric boundary conditions and loading are given in Fig. 1a, and an example 3D representation of the model is plotted in Fig. 1b with respect to the global coordinate basis $(x y z)$ and an illustrative discretisation using 419 tetrahedron elements. By applying symmetric boundary conditions at the $z^{-}$and $z^{+}$boundary surfaces, plane strain conditions are enforced. 
Thus, in the following, all results will be plotted in the $x y$-plane only. The rough footing interface condition is applied by setting the velocities in the $x$-direction to zero for all nodes in the footing region.

The obtained numerical upper bound results for different fineness of discretisation or degrees of freedom (DOF) are plotted in Fig. 2a. The black curve represents the results for continuous velocity fields, and shows clear convergence behaviour. Measuring the difference between the upper bound results and the analytical reference $N_{c}^{r e f}$, as $\operatorname{diff}(\%)=\left(N_{c}^{u b}-N_{c}^{r e f}\right) \cdot 100 /\left(N_{c}^{u b}+\right.$ $N_{c}^{r e f}$ ), a diff of $12.92 \%$ (2790 DOF) for point $a$ and $0.95 \%$ for point $c$ with 188685 DOF are obtained. The corresponding CPU times are 2.09 min versus $41.85 \mathrm{~min}$.

(a)

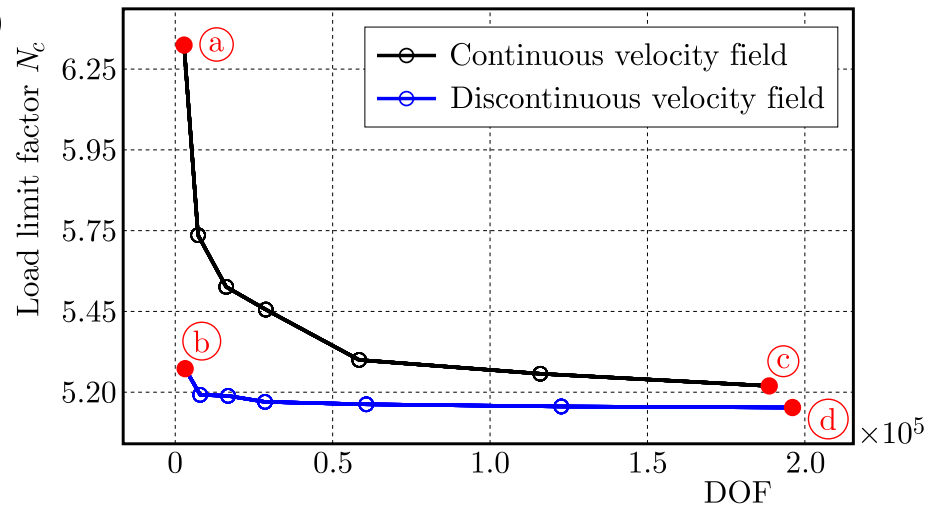

(b)

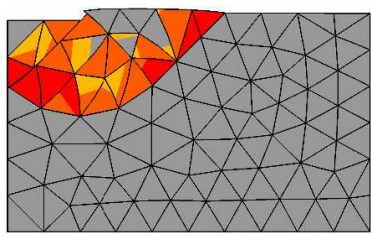

(c)

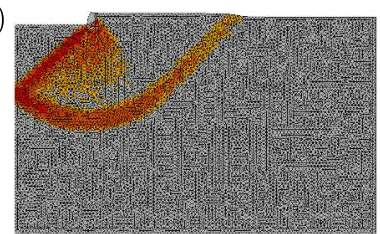

Fig. 2. Numerical upper bound results for the rigid strip footing problem: (a) load limit factor $N_{c}$ obtained using continuous and discontinuous velocity fields as a function of DOF; (b) upper bound failure mode using discontinuities and 445 elements (range of plotted yield function values $\left(\right.$ robjv) $\left.\left[-3 \cdot 10^{-8}: 0\right]\right) ;(\mathrm{c})$ upper bound failure mode using continuous velocity field with 31081 elements (robjv $\left.\left[-5 \cdot 10^{-9}: 0\right]\right)$

In the next step, partitions are introduced into the model according to Prandtl's failure mechanism (Prandtl, 1920), see Fig. 1c, to allow velocity jumps across prescribed discontinuities. Since velocity jumps are only allowed through these prescribed interfaces, the number of DOF is not increased significantly. The blue curve in Fig. 2a shows the related results, again for different numbers of DOF. A diff of $1.60 \%$ is obtained for point $b(3147 \mathrm{DOF})$ and $0.13 \%$ for point $d$ (196077 DOF), with corresponding CPU times of $0.56 \mathrm{~min}$ and $41.62 \mathrm{~min}$.

With a minimum difference of below $1 \%$ for the obtained best upper bounds compared to the analytical solution, the proposed formulations withstand this basic verification and can be assessed as performing well. Especially when velocity discontinuities are introduced, very good upper bounds can be obtained even with coarse meshes (see point $b$ and the related failure mechanism in Fig. 2b). Of course, this is only possible if the failure mechanism is known in advance and, thus, does not yet represent an added value for general calculations. However, the potential of velocity discontinuities to capture very localised failure is evidently huge and, sensibly used, can greatly increase computational efficiency.

\subsection{Block with asymmetric holes}

The block with asymmetric holes under tensile loading is a commonly-used benchmark for so-called direct methods (e.g. limit analysis and shakedown analysis) firstly studied by Zouain et al. (2002), as illustrated in Fig. 3a. Later on, this problem was studied by Makrodimopoulos and Martin (2007) using an upper bound formulation with a continuous quadratic velocity field, and the plane strain Mohr-Coulomb yield function was applied to the material. Their results are used for verification and as the reference solution in the following. Moreover, the benefit 
of using sensibly-arranged velocity discontinuities is further discussed, and a simple strategy to find such arrangements based on preliminary upper bound results is proposed. Geometry, material properties, and boundary conditions (see Fig. 3a) are assigned as in the reference (Makrodimopoulos and Martin, 2007). The model is built by 3D finite elements with the global coordinate basis $(x y z)$, similarly to Fig. 1b, and again symmetric boundary conditions at the $z-$ and $z+$ boundary surfaces are applied.

(a)

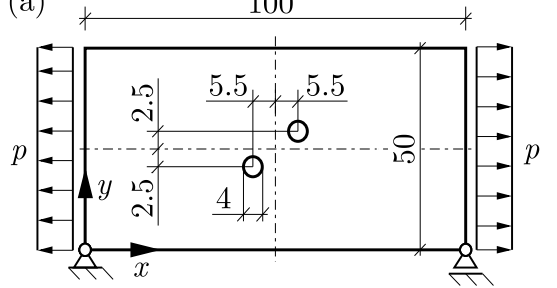

(c)

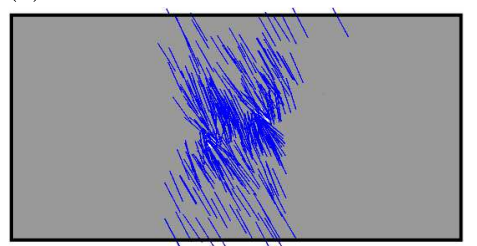

(d)

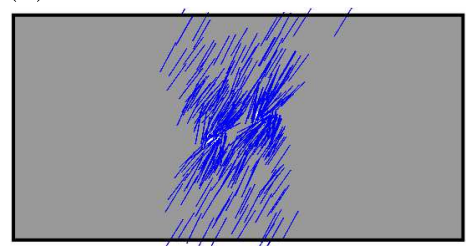

(b)

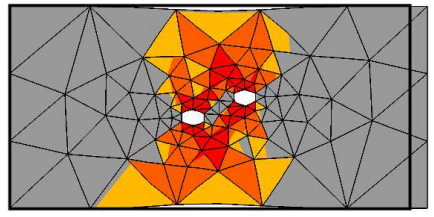

(e)

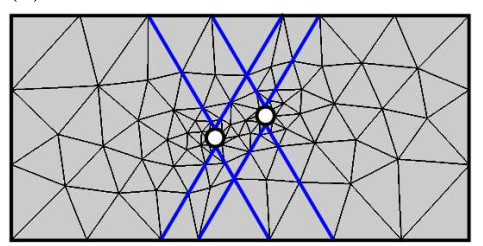

Fig. 3. Block with asymmetric holes using the Drucker-Prager failure criterion: (a) geometry and boundary conditions; (b) upper bound failure mode (519 elements, robjv $\left.\left[-5 \cdot 10^{-6}: 0\right]\right)$;

(c) and (d) potential orientations of plastic strain localisation; (e) prescribed discontinuities based on (c) and (d)

\subsubsection{Mohr-Coulomb failure criterion}

In Makrodimopoulos and Martin (2007), the Mohr-Coulomb yield function was used with a friction angle $\phi=30^{\circ}$. In our example, the equivalent Drucker-Prager yield function, $\sqrt{J_{2}} \leqslant A-B I_{1}$, is assigned to the material, defined by inserting

$$
\begin{aligned}
& \mathbf{P}_{\sigma}^{\text {mat }}=\frac{1}{3 A^{2}}\left[\begin{array}{cccccc}
1 & -0.5 & -0.5 & 0 & 0 & 0 \\
-0.5 & 1 & -0.5 & 0 & 0 & 0 \\
-0.5 & -0.5 & 1 & 0 & 0 & 0 \\
0 & 0 & 0 & 3 & 0 & 0 \\
0 & 0 & 0 & 0 & 3 & 0 \\
0 & 0 & 0 & 0 & 0 & 3
\end{array}\right] \quad \mathbf{F}_{\sigma}^{\text {mat },-}=\frac{1}{A^{2}}\left[\begin{array}{c}
2 A B \\
2 A B \\
2 A B \\
0 \\
0 \\
0
\end{array}\right] \\
& \mathbf{F}_{\sigma}^{\text {mat },+}=\mathbf{0}
\end{aligned}
$$

into Eq. (2.8), with the strength parameters $A=0.8321 c$ and $B=0.1601$. For the consistent traction-based yield function these terms read

$$
\mathbf{P}_{t}^{d i s}=\frac{1}{c^{2}}\left[\begin{array}{ccc}
-\tan ^{2} \phi & 0 & 0 \\
0 & 1 & 0 \\
0 & 0 & 1
\end{array}\right] \quad \mathbf{F}_{t}^{d i s,+}=\mathbf{0} \quad \mathbf{F}_{t}^{d i s,-}=\frac{1}{c^{2}}\left[\begin{array}{c}
2 c \tan \phi \\
0 \\
0
\end{array}\right]
$$

In the previous example, it has been shown that velocity discontinuities can improve the upper bound significantly if they are appropriately arranged with respect to potential failure surfaces. For this reason, subsequently, the following strategy is pursued. Based on an upper bound calculation with a continuous velocity field and a relatively coarse mesh, as shown in 
Fig. 3b, potential discontinuity directions, where plastic failure could localise, are computed at each integration point where plastic flow takes place according to Eq. (2.10). The resulting orientations are plotted in Fig. 3c and Fig. 3d. With respect to the $x$ axis, the mean orientations obtained by taking the volume average over all orientations are approximately $\bar{\theta}_{1}=-60^{\circ}$ and $\bar{\theta}_{2}=60^{\circ}$, as would be expected from the underlying failure criterion. Next, according to these average directions of possible localised plastic failure, and by referring to the points with maximum plastic strain-rates, the partitions (blue lines) shown in Fig. 3e are implemented into the model. Finally, the model is re-meshed and velocity discontinuities are introduced along the partitions.

This procedure has been applied to several models with different level of mesh refinement (DOF) and compared to calculations performed without such discontinuities. The obtained upper bounds for the limit load factor $N_{c}$ are plotted in Fig. 4a. Based on the upper bound result with only 3327 DOF (point $a$ ), velocity discontinuities were introduced into the model, leading to a strong improvement of the upper bound (point $b$ ) by adding only 585 DOF. The failure mechanism associated with point $b$ is shown in Fig. $4 \mathrm{~b}$. To achieve a similarly good upper bound result and the related localised failure mechanism without the introduction of discontinuities, the mesh needed to be refined significantly, as shown in Fig. 4c as the associated failure mechanism to point $c$. The CPU time required to obtain point $c$ and the mechanism in Fig. 4c was 37.87 min, whereas point $b$ with the mechanism shown in Fig. 4 b only took 0.38 min. Although an adaptive mesh refinement would probably be more efficient than the uniform mesh refinement performed, the performance of sensibly-arranged discontinuities, even in a coarsely discretised model, is excellent. Comparing the upper bound result of point $b\left(N_{c}=1.062\right)$ to the best result in the reference (Makrodimopoulos and Martin, 2007) $\left(N_{c}=1.063\right)$ there is almost no difference. However, the upper bound indicated by point $b$ was obtained with 3912 DOF compared to 79955 DOF in the reference.

(a)

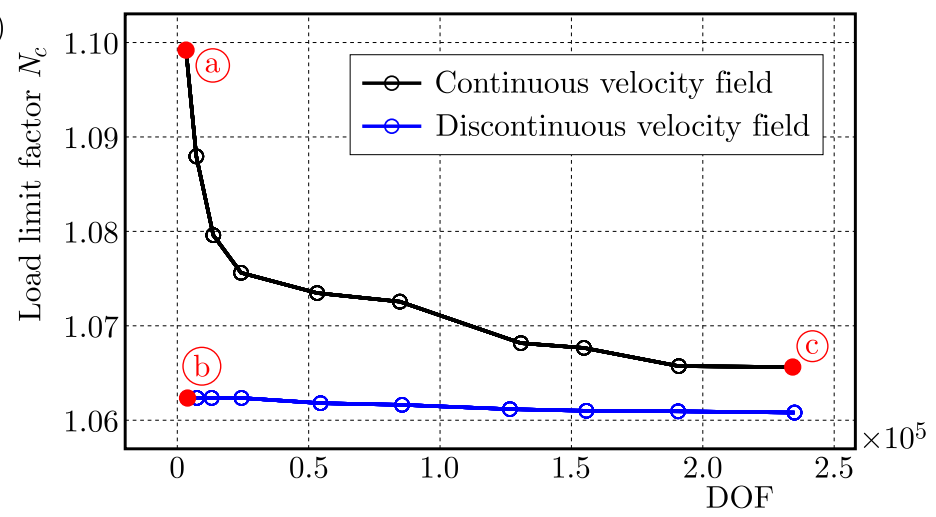

(b)

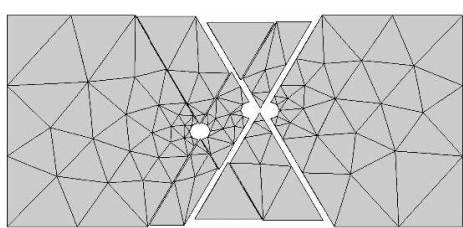

(c)

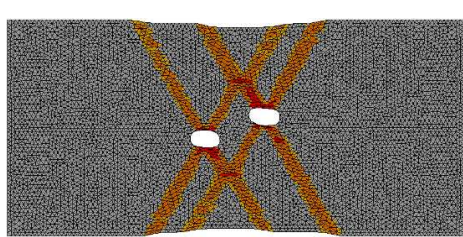

Fig. 4. Numerical upper bound results for the block with asymmetric holes example using the Drucker-Prager yield function: (a) load limit factor $N_{c}$ obtained using continuous and discontinuous velocity fields as a function of DOF; upper bound results (robjv $\left[-1 \cdot 10^{-4}: 0\right]$ ) (b) with discontinuities using 504 elements and (c) with continuous velocity field using 34875 elements

In the following, this approach is extended to orthotropic material behaviour, using the Tsai-Wu criterion as the indicator for plastic failure.

\subsubsection{Tsai-Wu failure criterion}

The strength parameters used for the example correspond to the typical orthotropic material spruce wood and are taken from (Dorn, 2012), leading to 


$$
\begin{aligned}
& \mathbf{P}_{\sigma}^{\text {mat }}=\left[\begin{array}{cccccc}
2.434 \mathrm{E}-4 & 0 & 0 & 0 & 0 & 0 \\
0 & 6.588 \mathrm{E}-2 & 0 & 0 & 0 & 0 \\
0 & 0 & 6.588 \mathrm{E}-2 & 0 & 0 & 0 \\
0 & 0 & 0 & 1.181 \mathrm{E}-2 & 0 & 0 \\
0 & 0 & 0 & 0 & 2.973 \mathrm{E}-2 & 0 \\
0 & 0 & 0 & 0 & 0 & 1.181 \mathrm{E}-2
\end{array}\right] \mathrm{MPa}^{-2} \\
& \mathbf{F}_{\sigma}^{\text {mat },+}=\mathbf{F}_{\sigma}^{\text {mat },-}=\left[\begin{array}{llllll}
-6.573 \mathrm{E}-3 & 8.564 \mathrm{E}-2 & 8.564 \mathrm{E}-2 & 0 & 0 & 0
\end{array}\right]^{\mathrm{T}} \mathrm{MPa}^{-1}
\end{aligned}
$$

inserted into the general yield function in Eq. (2.8), defined with respect to the local coordinate basis $(L T R)$. Due to the orthotropic characteristics, the consistent traction-based yield function is highly dependent on the orientation of the discontinuity at which it describes plastic failure. Thus, for each introduced discontinuity, a different set of strength parameters had to be computed, determined by the "projection" procedure introduced at the end of Section 2. Then, the equivalence between the material strength within elements and the strength behaviour at discontinuities is guaranteed.

In the following, this orthotropic strength behaviour is assigned to the block with asymmetric holes, for two different material orientations, to assess the capability of the presented approach in handling anisotropic strength behaviour.

\section{$\underline{\text { Material orientation } 1}$}

In the first case, the longitudinal orientation $L$ of the material is identical to the $y$-direction, as indicated in Fig. 5a. Moreover, the local material coordinate basis (LTR) is defined with $L$ and $T$ as the in-plane axes and $R$ as the out-of-plane axis.

(a)

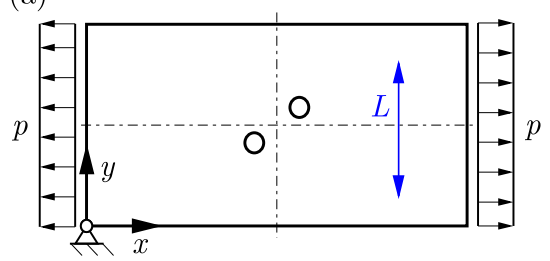

(c)

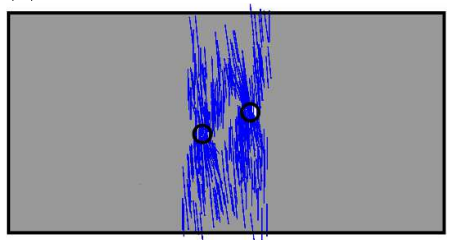

(b)

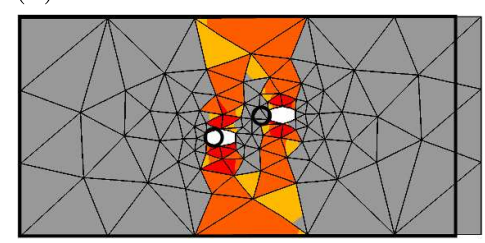

(e)

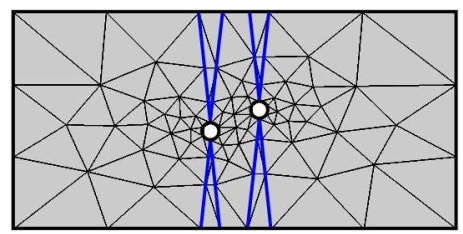

Fig. 5. Block with asymmetric holes using the Tsai-Wu orthotropic failure criterion: (a) geometry, boundary conditions, and the principal material orientation indicated by the blue arrow; (b) upper bound result using 519 elements (robjv $\left[-1.5 \cdot 10^{-6}: 0\right]$ ); (c) and (d) potential orientations of plastic strain localisation; (e) prescribed discontinuities based on (c) and (d)

The preliminary upper bound calculation, based on which the arrangement of discontinuities will be defined, is carried out using a coarse mesh with 519 elements. The corresponding failure mechanism is displayed in Fig. 5b, and the computed orientations of potential discontinuities are shown in Figs. 5c and 5d. Averaging over these orientations results in two mean angle values of $\bar{\theta}_{1}=-84^{\circ}$ and $\bar{\theta}_{2}=84^{\circ}$ with respect to the $x$-axis. According to these mean angles and starting from the points of highest plastic strain-rates (present at the boundaries of the holes), the velocity discontinuities shown in Fig. 5e are implemented into the model. Subsequently, the model is re-meshed and a further upper bound calculation is performed. 
(a)

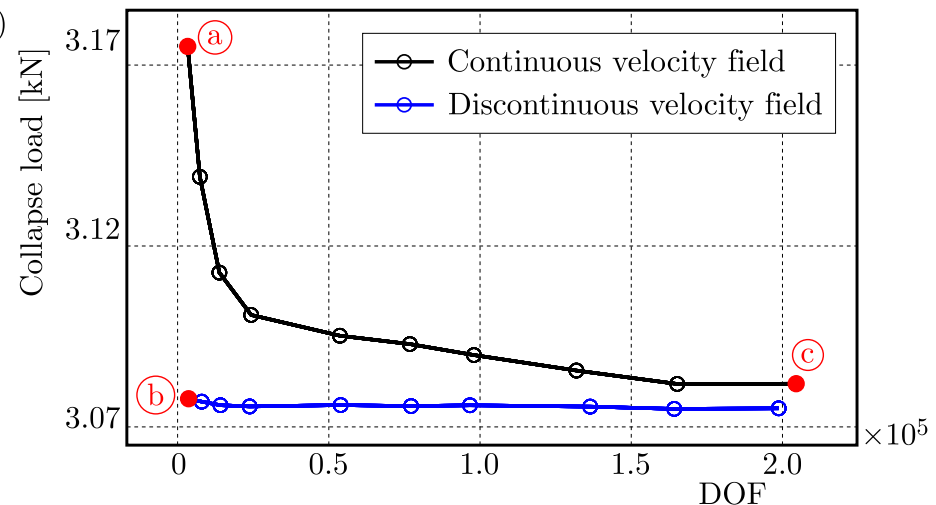

(b)

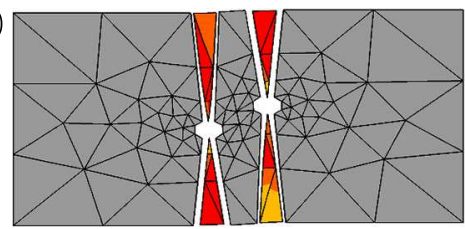

(c)

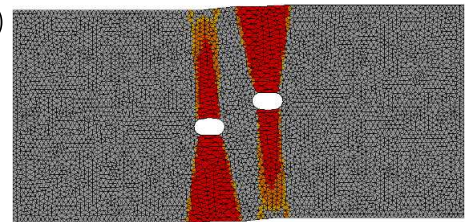

Fig. 6. Numerical upper bound results for the block with asymmetric holes with the principal material direction as defined in Fig. 5a: (a) collapse load using continuous and discontinuous velocity fields as a function of DOF; upper bound failure mode (robjv $\left[-9 \cdot 10^{-6}: 0\right]$ ) using (b) discontinuities within a coarse mesh (486 elements) and (c) a continuous velocity field with a fine mesh (38537 elements)

The numerical upper bounds on the collapse load for different levels of mesh refinement (DOF) with and without discontinuities are plotted in Fig. 6. Again, the introduction of velocity discontinuities improves the upper bound significantly, while hardly increasing the DOF. In contrast to the isotropic case before, plastic failure also occurs in the solid finite elements between discontinuities (see Fig. 6b), indicating that the orientation or arrangement of discontinuities could be improved. This can also be seen by looking at the plastic regions in Fig. 6c, which do not exactly match the definition of discontinuities above. It seems that the arrangement of discontinuities does not necessarily have to be ideal in order to achieve considerable improvement in the numerical upper bound results.

\section{Material orientation 2}

In the second case, the local material orientation basis $(L T R)$ is rotated by $30^{\circ}$ in the $x y$ plane, as indicated in Fig. 7a. Again, based on an efficient preliminary upper bound calculation (see Fig. 7b), possible orientations of discontinuities are computed, resulting in mean angles of $\bar{\theta}_{1}= \pm 80^{\circ}$ and $\bar{\theta}_{2}=-58^{\circ}$. The introduced velocity discontinuities are shown in Fig. 7e.

(a)

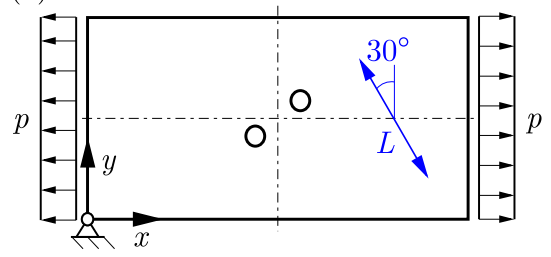

(d)

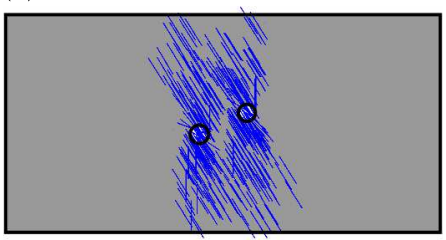

(b)

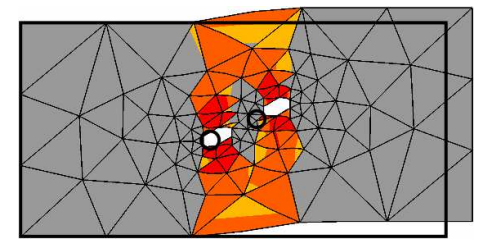

(e)

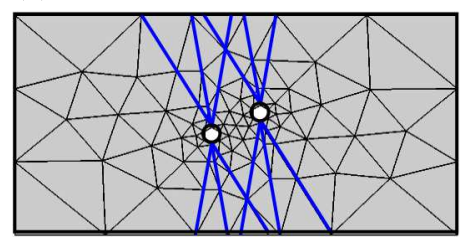

Fig. 7. Block with asymmetric holes using the Tsai-Wu orthotropic failure criterion: (a) geometry, boundary conditions, and the principal material orientation indicated by the blue arrow; (b) upper bound result using 519 elements (robjv $\left[-3 \cdot 10^{-6}: 0\right]$ ); (c) and (d) potential orientations of plastic strain localisation; (e) prescribed discontinuities based on (c) and (d) 
(a)

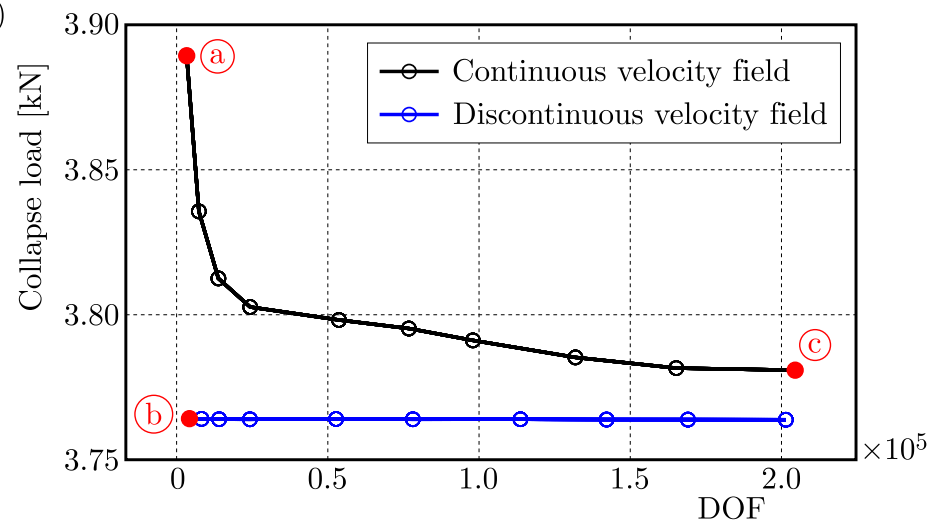

(b)

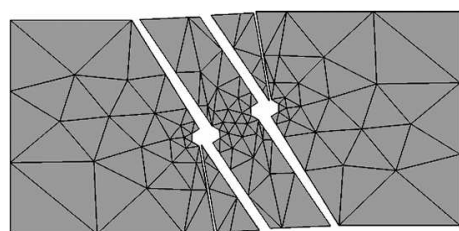

(c)

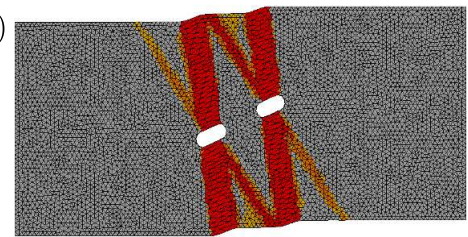

Fig. 8. Numerical upper bound results for the block with asymmetric holes with the principal material direction as defined in Fig. 7a: (a) collapse load using continuous and discontinuous velocity fields as a function of DOF; upper bound failure mode (robjv $\left[-2 \cdot 10^{-5}: 0\right]$ ) using (b) discontinuities within a coarse mesh (524 elements) and (c) a continuous velocity field with a fine mesh (34875 elements)

As before, all numerical upper bounds are plotted in Fig. 8, showing the strong performance increase achieved by the selectively introduced discontinuities. Interestingly, the intensity of localisation of plastic failure is slightly different comparing the approaches with (Fig. 8b) and without (Fig. 8c) discontinuities. It seems that with discontinuities the real failure mechanism can be better represented, since the main plastic failure direction is well aligned with the principal material direction (wood fibre direction) as expected.

\subsection{Shear test on block}

The last example is used to assess the capability of this upper bound formulation regarding localised shear failure in orthotropic materials. The setup of the model is shown in Fig. 9a and is designed to represent a characteristic as well as often critical loading states in wood-based products, like glued-laminated timber and cross-laminated timber. As in the previous examples, the boundary conditions are chosen so as to represent plane strain conditions, and the Tsai-Wu failure criterion with strength parameters representing spruce wood is assigned to the material.

Again, based on a preliminary upper bound calculation using a very coarse mesh (Fig. 9c1), the orientations of possible discontinuities are determined (Fig. 9c2) and, based on that information, velocity discontinuities are implemented into the model (Fig. 9c3). The result obtained with this discontinuity arrangement is shown in Fig. 9d1, where the dominant plastic failure appears within the solid elements between the introduced discontinuities but not at the discontinuities themselves, and, thus, the potential of the velocity discontinuities has not been activated sufficiently. For this reason, a second iteration was carried out, again computing the orientations of possible discontinuities in all plastic regions (Fig. 9d2). Based on that information, a revised discontinuity pattern was implemented as shown in Fig. 9d3. The corresponding failure mechanism is displayed in Fig. 10b and it can be seen that, now, localised failure occurs exclusively at the last-introduced discontinuity. The related upper bound (point $b$ in Fig. 10a) is very good in comparison to the preliminary model without discontinuities (point $a$ ) but uses a similar number of DOF. Moreover, the failure mechanism agrees well with that obtained using a very fine mesh and no discontinuities (Fig. 10c). 
(a)

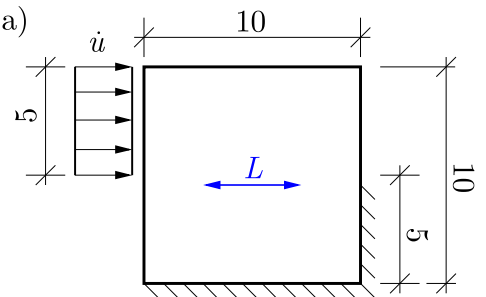

(c1)

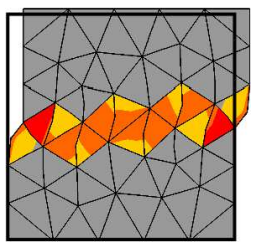

(d1)

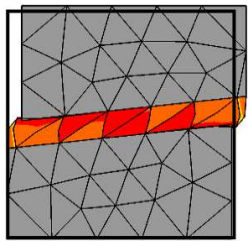

(c2)

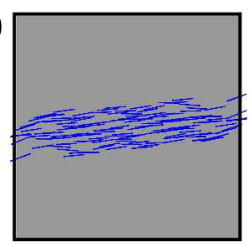

(d2)

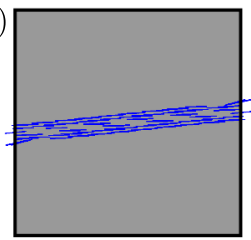

(b)

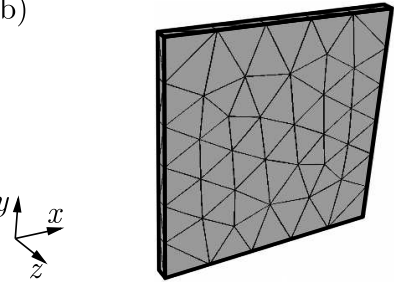

(c3)

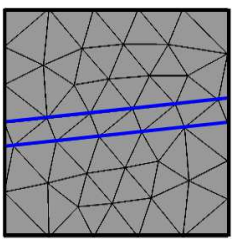

(d3)

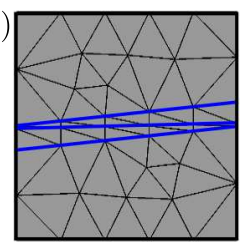

Fig. 9. Block under shear loading: (a) geometry, boundary conditions and the principal material orientation indicated by the blue arrow; (b) illustrative 3D model with 222 finite elements;

(c) and (d) two iteration steps for the definition of the discontinuities, with (c1) and (d1) as the upper bound result (robjv $\left.\left[-2 \cdot 10^{-9}: 0\right]\right),(\mathrm{c} 2)$ and $(\mathrm{d} 2)$ as the potential orientations of plastic strain localisation, and (c3) and (d3) as the prescribed discontinuities based on (c2) and (d2)

(a)

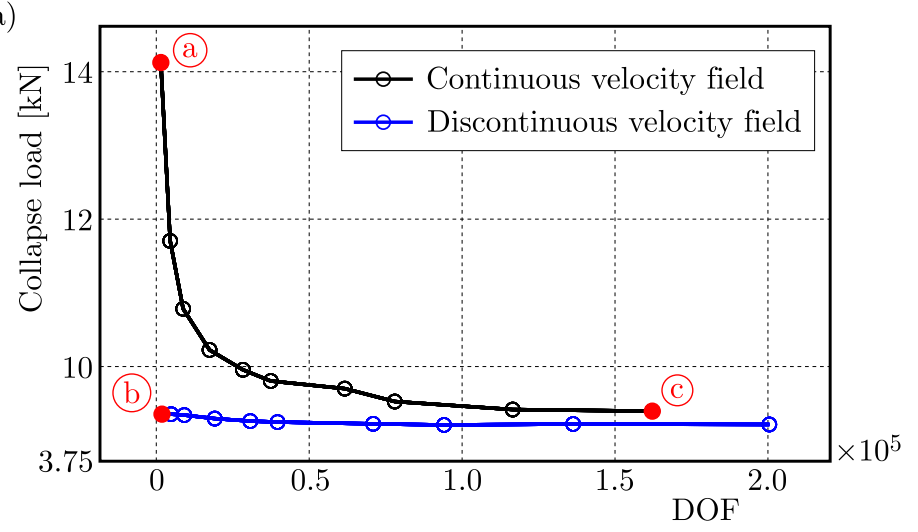

(b)

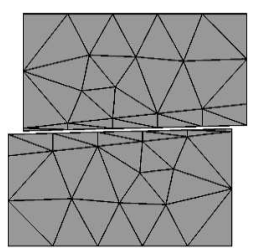

(c)

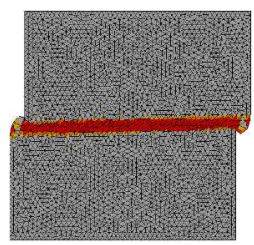

Fig. 10. Numerical upper bound results for the block under shear loading: (a) collapse load using continuous and discontinuous velocity fields as a function of DOF; the upper bound failure mode (robjv

$\left.\left[-4 \cdot 10^{-9}: 0\right]\right)$ using (b) discontinuities within a coarse mesh (242 elements) and (c) a continuous velocity field with a fine mesh (32062 elements)

\section{Summary and conclusions}

In this work, a 3D numerical upper bound formulation using a quadratic approximation of the velocity field and allowing for the implementation of orthotropic yield functions has been briefly proposed. Furthermore, the implementation of velocity discontinuities into this formulation has been presented, along with the concept of how to derive the necessary traction-based yield function which guarantees a consistent description of the material strength behaviour across discontinuities. Based on that formulation, compatibility as well as the associated plastic flow rule are fulfilled throughout the whole body and, thus, rigorous upper bounds are obtained. 
The formulation has been verified by means of two classical examples, the rigid strip footing and the block with asymmetric holes. Subsequently, based on preliminary upper bound calculations with very coarse meshes, the orientation of potential slip lines where very localised plastic flow may occur could be determined. Based on that information, sensibly-arranged velocity discontinuities were incorporated into the finite element models. In that way, upper bounds could be improved significantly with essentially no increase of degrees of freedom.

It has also been shown that this concept works very well when assigning orthotropic failure behaviour to the material. If the first introduction of discontinuities does not improve the upper bound significantly, which means that most of the plastic dissipation still takes place in solid elements, a second iteration step can improve the situation, as shown by means of the shear test on a block. This may represent an important finding for future developments, which could lead to a general algorithm for an adaptive introduction and re-arrangement of velocity discontinuities, as an efficient alternative to existing adaptive mesh refinement strategies. Especially for laminated structures and orthotropic materials, where plastic failure often occurs in a very localised mechanism, as shown for wood at the microscale in (Lukacevic et al., 2014; Lukacevic and Füssl, 2016) and at the product scale in (Hochreiner et al., 2013, 2014), such an approach could have great value. Moreover, the efficiency of numerical limit analysis in combination with the accuracy of the extended finite element formulations presented in (Lukacevic et al., 2014; Lukacevic and Füssl, 2016) could lead to more flexible engineering design tools, in which the focus can be switched between accuracy and efficiency as needed.

\section{Acknowledgments}

Financial support for this work in the framework of the PhDSchool DokIn'Holz funded by the Austrian Federal Ministry of Science, Research and Economy and the Austrian Association of Wood Industries is gratefully acknowledged. We also gratefully acknowledge the financial support of this work by the Austrian Science Fund (FWF) through the Erwin Schrödinger Fellowship J3748-N30.

\section{References}

1. Anderheggen E., KnöPfel H., 1972, Finite element limit analysis using linear programming, International Journal of Solids and Structures, 8, 12, 1413-1431

2. Andersen E.D., Roos C., Terlaky T., 2003, On implementing a primal-dual interior-point method for conic quadratic optimization, Mathematical Programming, 95, 2, 249-277

3. Belytschko T., Hodge P.G., 1970, Plane stress limit analysis by finite elements, Journal of the Engineering Mechanics Division, 96, 6, 931-944

4. Bottero A., Negre R., Pastor J., Turgeman S., 1980, Finite element method and limit analysis theory for soil mechanics problems, Computer Methods in Applied Mechanics and Engineering, 22, 1, 131-149

5. Chen J., Yin J.-H., Lee C.F., 2003, Upper bound limit analysis of slope stability using rigid finite elements and nonlinear programming, Canadian Geotechnical Journal, 40, 742-752

6. Ciria H., Peraire J., 2004, Computation of upper and lower bounds in limit analysis using second-order cone programming and mesh adaptivity, 9th ASCE Specialty Conference on Probabilistic Mechanics and Structural Reliability

7. Ciria H., Peraire J., Bonet J., 2008, Mesh adaptive computation of upper and lower bounds in limit analysis, International Journal for Numerical Methods in Engineering, 75, 8, 899-944

8. Dorn M., 2012, Investigations on the Serviceability Limit State of Dowel-Type Timber Connections, $\mathrm{PhD}$ Thesis, Vienna University of Technology

9. Drucker D.C., Greenberg H.J., Prager W., 1951, The safety factor of an elastic-plastic body in plane strain, Journal of Applied Mechanics, 18, 371-378 
10. Drucker D.C., Prager W., Greenberg H.J., 1952, Extended limit design theorems for continuous media, Quarterly of Applied Mathematics, 9, 381-389

11. Füssl J., Lackner R., Eberhardsteiner J., Mang H.A., 2008, Failure modes and effective strength of two-phase materials determined by means of numerical limit analysis, Acta Mechanica, 1-4, 195, 185-202

12. Füssl J., Li M., Lukacevic M., Eberhardsteiner J., Martin C.M., 2017, Comparison of unit cell-based computational methods for predicting the strength of wood, Engineering Structures, 141, 427-443

13. Hawksbee S., Smith C., Gilbert M., 2013, Application of discontinuity layout optimization to three-dimensional plasticity problems, Proceedings of the Royal Society of London A: Mathematical, Physical and Engineering Sciences, 469, 2155

14. Hochreiner G., Füssl J., Eberhardsteiner J., 2013, Cross-laminated timber plates subjected to concentrated loading - experimental identification of failure mechanisms, Strain, 50, 1, 68-81

15. Hochreiner G., Füssl J., Serrano E., Eberhardsteiner J., 2014, Influence of wooden board strength class on the performance of cross-laminated timber plates investigated by means of full-field deformation measurements, Strain, 50, 2, 161-173

16. Krabbenhøft K., Lyamin A.V., Huiaj M., Sloan S.W., 2005, A new discontinuous upper bound limit analysis formulation, International Journal for Numerical Methods in Engineering, 63, 7, 1069-1088

17. Le C.V., Askes H., Gilbert M., 2019, Adaptive element-free Galerkin method applied to the limit analysis of plates, Computer Methods in Applied Mechanics and Engineering, 199, 37, $2487-2496$

18. Li M., Füssl J., Lukacevic M., Eberhardsteiner J., Martin C.M., 2018, Strength predictions of clear wood at multiple scales using numerical limit analysis approaches, Computers and Structures, 196, 200-216

19. LiU F., Zhaо J., 2013, Upper bound limit analysis using radial point interpolation meshless method and nonlinear programming, International Journal of Mechanical Sciences, 70, 26-38

20. LukACEVIC M., Füssl J., 2016, Application of a multisurface discrete crack model for clear wood taking into account the inherent microstructural characteristics of wood cells, Holzforschung, 70, $9,845-853$

21. Lukacevic M., Füssl J., LAmpert R., 2014, Failure mechanisms of clear wood identified at wood cell level by an approach based on the extended finite element method, Engineering Fracture Mechanics, 144, 158-175

22. Lyamin A.V., Sloan S.W., 2002a, Lower bound limit analysis using non-linear programming, International Journal for Numerical Methods in Engineering, 55, 5, 573-611

23. Lyamin A.V., Sloan S.W., 2002b, Upper bound limit analysis using linear finite elements and non-linear programming, International Journal for Numerical and Analytical Methods in Geomechanics, 26, 2, 181-216

24. LySMER J., 1970, Limit analysis of plane problems in soil mechanics, Journal of the Soil Mechanics and Foundations Division, 96, 4, 1311-1334

25. Maier G., Zavelani-Rossi A., Benedetti D., 1972, A finite element approach to optimal design of plastic structures in plane stress, International Journal for Numerical Methods in Engineering, 4, 4, 455-473

26. Makrodimopoulos A., Martin C.M., 2006, Lower bound limit analysis of cohesive-frictional materials using second-order cone programming, International Journal for Numerical Methods in Engineering, 66, 4, 604-634

27. Makrodimopoulos A., Martin C.M., 2007, Upper bound limit analysis using simplex strain elements and second-order cone programming, International Journal for Numerical and Analytical Methods in Geomechanics, 31, 6, 835-865 
28. Makrodimopoulos A., Martin C.M., 2008, Upper bound limit analysis using discontinuous quadratic displacement fields, Communications in Numerical Methods in Engineering, 24, 11, 911-927

29. Martin C., Makrodimopoulos A., 2008, Finite-element limit analysis of Mohr-Coulomb materials in 3D using semidefinite programming, Journal of Engineering Mechanics, 134, 4, 339-347

30. Milani G., Lourenço P.B., 2009, A discontinuous quasi-upper bound limit analysis approach with sequential linear programming mesh adaptation, International Journal of Mechanical Sciences, 51, 89-104

31. MOSEK ApS, 2014, The MOSEK optimization tools version 7.0, User's Manual and Reference, http://www.mosek.com

32. PrandTL L., 1920, Über die Härte plastischer Körper, Nachrichten von der Gesellschaft der Wissenschaften zu Göttingen, Mathematisch-Physikalische Klasse, 12, 74-85

33. Sloan S.W., Kleeman P.W., 1995, Upper bound limit analysis using discontinuous velocity fields, Computer Methods in Applied Mechanics and Engineering, 127, 14, 293-314

34. Smith C., Gilbert M., 2007, Application of discontinuity layout optimization to plane plasticity problems, Proceedings of the Royal Society A, 463, 2461-2484

35. Wu J.-Y., Cervera M., 2014, On the equivalence between traction- and stress-based approaches for the modeling of localized failure in solids, Journal of the Mechanics and Physics of Solids, 82, 137-163

36. Yu S., Zhang X., Sloan S.W., 2016, A 3D upper bound limit analysis using radial point interpolation meshless method and second-order cone programming, International Journal for Numerical Methods in Engineering, 108, 13, 1686-1704

37. Zouain N., Borges L., Silveira J.L., 2002, An algorithm for shakedown analysis with nonlinear yield functions, Computer Methods in Applied Mechanics and Engineering, 191, 23, 2463-2481 